\title{
Les dernières heures du Christ sur la croix " racontées » en photographie: The Seven Words (1898) de Fred Holland Day
}

Nathalie Dietschy

\section{OpenEdition}

Journals

Édition électronique

URL : http://journals.openedition.org/edl/585

DOI : $10.4000 /$ edl.585

ISSN : 2296-5084

Éditeur

Université de Lausanne

\section{Édition imprimée}

Date de publication : 15 décembre 2013

Pagination : 223-244

ISBN : 978-2-940331-33-8

ISSN : 0014-2026

\section{Référence électronique}

Nathalie Dietschy, «Les dernières heures du Christ sur la croix « racontées » en photographie: The Seven Words (1898) de Fred Holland Day ", Études de lettres [En ligne], 3-4 | 2013, mis en ligne le 15 décembre 2016, consulté le 18 décembre 2020. URL : http://journals.openedition.org/edl/585 ; DOI : https://doi.org/10.4000/edl.585 


\section{LES DERNIÈRES HEURES DU CHRIST SUR LA CROIX "RACONTÉES" EN PHOTOGRAPHIE: THE SEVEN WORDS (1898) DE FRED HOLLAND DAY}

En 1900, le critique Sadakichi Hartmann écrit que le photographe américain Fred Holland Day, au travers de ses clichés, conte des histoires. Pourtant, la capacité de la photographie à «raconter» n'est pas unanimement admise, aujourd'hui encore. L'image fixe - photographie comme peinture - est confrontée à la difficulté de traduire dans l'espace l'idée de temporalité. Nous souhaiterions analyser ici la séquence photographique de Day, The Seven Words (1898), qui interroge directement la capacité de la photographie à raconter une histoire, celle de la vie de Jésus, du moins les dernières heures vécues sur la croix. Nous postulons que le choix de ce sujet relève de la volonté du photographe de prouver les qualités de son médium, égal à la peinture, voire à la poésie...

Pictorial representation of a classic subject on classic lines has spoken its first word in artistic photography, and no one knows where it may lead to.

Sadakichi Hartmann, "Portrait Painting and Portrait Photography» [1899] ${ }^{1}$

\section{Narration et image fixe}

«La photographie [...] n'eut jamais le projet de conter des histoires. Quand elle le fait, c'est qu'elle imite le cinéma: elle étale dans l'espace la successivité que le film aurait déployée dans le temps » ${ }^{2}$. Par ces mots, Christian Metz refuse à la photographie la faculté de raconter

I. In S. Hartmann, The Valiant Knights of Daguerre, p. 48.

2. Ch. Metz, «Le cinéma», p. 63. 
une histoire, idée qu'il n'est pas le seul à défendre ${ }^{3}$. L'image fixe - photographie comme peinture - est confrontée à la difficulté de traduire dans l'espace l'idée de temporalité ${ }^{4}$. Mais si on admet que la peinture peut organiser spatialement des zones temporelles distinctes (l'arrièreplan correspondant au passé et le premier plan au présent par exemple), "la photographie a du mal à échapper à la simultanéité de sa prise de vue ${ }^{5}$. Le rapport au réel qu'entretient la photographie, sa relation directe au référent - le "ça a été " ${ }^{6}$ formulé par Roland Barthes -, l'impression d'instantané qu'elle procure paraissent s'opposer à la possibilité d'exprimer une durée et de développer un récit.

Pourtant, en 1900, le critique Sadakichi Hartmann, décrivant l'œuvre du photographe américain Fred Holland Day (1864-1933), octroie à ce dernier les capacités d'un véritable conteur («complete story-telling») 7 . Nous souhaiterions donc analyser ici une séquence photographique réalisée par Day - son chef-d'œuvre - création qui interroge directement la capacité de la photographie à raconter une histoire et en particulier, le récit de la vie de Jésus.

\section{La fonction du cadre}

En 1898, le photographe Fred Holland Day - également éditeur ${ }^{8}$ et collectionneur de livres - réalise plus de 250 négatifs représentant les scènes marquantes de la vie de Jésus. Une œuvre se démarque des autres clichés réalisés cette année-là: The Seven Words, connue aussi sous le titre The Seven Last Words of Christ (1898, fig. 1) ${ }^{9}$. Celle-ci a été prise dans un

3. J.-M. Schaeffer, «Narration visuelle et interprétation».

4. Analysant les œuvres d'art antiques, Kurt Weitzmann dénombre trois modes de narrativité visuelle: la méthode simultanée, monoscénique et cyclique. K. Weitzmann, Illustration in Roll and Codex, p. 13 sq.

5. C. Scott, The Spoken Image, p. 244.

6. R. Barthes, La Chambre claire, p. 120.

7. «F. Holland Day. A Decorative Photographer» [1900], in S. Hartmann, The Valiant Knights of Daguerre, p. 186.

8. Fred Holland Day co-fonde la maison d'édition Copeland and Day. Voir P. J. Fanning, Through an Uncommon Lens.

9. Epreuves au platine, ca $14 \times 11,5 \mathrm{~cm}$ chaque tirage, New York, The Metropolitan Museum of Art. 
Fig. 1 - Fred Holland Day, Photographie de la séquence The Seven Words, 1898.

décor intérieur ${ }^{10}$ et se compose de sept tirages de format identique exposés généralement comme un ensemble homogène et clos, même si Day a également présenté certains tirages comme des œuvres autonomes ${ }^{11}$.

Plusieurs cadres ont été employés pour présenter The Seven Words. L'un, noir laqué, réunit les sept tirages en frise ${ }^{12}$. Le cadre, qui rappelle par son format les prédelles des retables chrétiens, sépare chaque épreuve par une fine bordure qui à la fois délimite les compartiments, tout en créant une relation d'interdépendance entre les photographies. Une seconde version existe ${ }^{13}$ : sur la partie inférieure du cadre, sous chaque tirage correspondant, sont inscrites les sept dernières paroles du Christ rapportées dans les évangiles, "Father forgive them; they don't know what they do", "Today thou shalt be with me in paradise", "Woman, behold thy son; son, thy mother", "My God, my God, why hast thou forsaken me?", "I thirst", "Into thy hands I commend my spirit", "It is finished». Sur la partie supérieure du cadre trônent le titre original The Seven Words, ainsi que l'auteur et la provenance de l'œuvre: "By F. H. Day, Boston, Mass., Given by Mr. Clarence B. Moore». Un dernier cadre est connu, plus élaboré, composé de pilastres de chapiteaux corinthiens (fig. 1). Il semble qu'il ait été utilisé pour les publications de l'œuvre ${ }^{14}$, mais une prise de vue de l'exposition American Pictorial Photography organisée par le photographe américain Alfred Stieglitz au National Arts Club de New York en 1902, plaide en faveur d'une

Io. V. Posever Curtis, «F. Holland Day», p. 311.

II. Voir ibidem.

I2. Une reproduction se trouve in P. J. Fanning, Through an Uncommon Lens. Collection du Museum of Fine Arts de Boston.

I3. Pour une reproduction, voir Art 39 Basel, p. 503.

I4. J. Crump, "F. Holland Day: "Sacred" Subjects and "Greek Love”", p. 325; V. Posever Curtis, «F. Holland Day», note 87. 
utilisation de ce cadre imposant également lors d'expositions ${ }^{15}$. Les inscriptions ne se situent plus sous chaque épreuve, mais au-dessus et à la suite les unes des autres, détachées de l'image à laquelle chaque parole est associée. La place du texte manifeste d'ailleurs le rôle de l'écrit au sein de l'œuvre: les mots n'empiètent jamais l'espace de l'image; au contraire ils la soulignent, l'accompagnent, la présentent.

La fonction du cadre est ici fondamentale ${ }^{16}$. Non seulement il "sépare l'image de tout ce qui est non-image ${ }^{17}$, mais sa structure imposante signale la valeur autoritaire de l'œuvre photographique qui y est déployée. Lorsque le photographe envoie les Seven Words au Salon photographique de Philadelphie en 1898, il explique dans une lettre à Stieglitz: "Il y a un cadre contenant sept tirages dans l'ordre d'une séquence à laquelle j'ai pris la liberté de donner un titre...» ${ }^{18}$. Fred Holland Day conçoit bien cette suite de sept photographies comme un ensemble narratif, une "séquence», selon ses propres mots ${ }^{19}$. Pour qu'une image fixe traduise la succession temporelle, soit il y a "coprésence» - c'est l'image à scènes multiples -, soit il y a "contiguïté spatiale ${ }^{20}$, comme dans les séries. Mais alors que la série peut se définir comme «une succession continue ou discontinue d'images liées par un

15. Une reproduction de la vue de l'exposition se trouve in K. Schwain, «F. Holland Day's Seven Last Words and the Religious Roots of American Modernism ", p. 36. L'auteure affirme d'ailleurs que le cadre à l'architecture classique a aussi été utilisé à Paris et à Londres en 1900.

I6. Sur le type d'encadrement que choisit Day pour exposer ses photographies conçues comme des œuvres d'art uniques et sur l'influence du mouvement Arts and Crafts qui valorise les arts appliqués, voir: V. Posever Curtis, «F. Holland Day», p. 311.

17. V. I. Stoichita, L'Instauration du tableau, p. 53.

I8. "There is one frame which contains seven prints in sequence which I have taken the liberty of calling one title...». Notre traduction (idem pour les prochaines occurrences). Lettre de Fred Holland Day à Stieglitz, septembre 1898, Stieglitz Archives, cité in The Photographic Work of F. Holland Day, p. 30. Un cadre noir est choisi pour la première exposition de l'œuvre, au Salon photographique de Philadelphie en 1898. K. Schwain, "F. Holland Day's Seven Last Words and the Religious Roots of American Modernism», p. 48.

I9. La séquence photographique est généralement formée d'images de format identique, chacune dans un cadre propre. Voir J. Baetens, M. Bleyen, «Photo Narrative, Sequential Photography, Photonovels». Patricia J. Fanning décrit l'œuvre en utilisant le terme de «narration»: "[...] a sequence of seven photographs visually narrating the final phrases spoken by Christ». P. J. Fanning, Through an Uncommon Lens, p. 107.

20. J.-M. Schaeffer, «Narration visuelle et interprétation». 


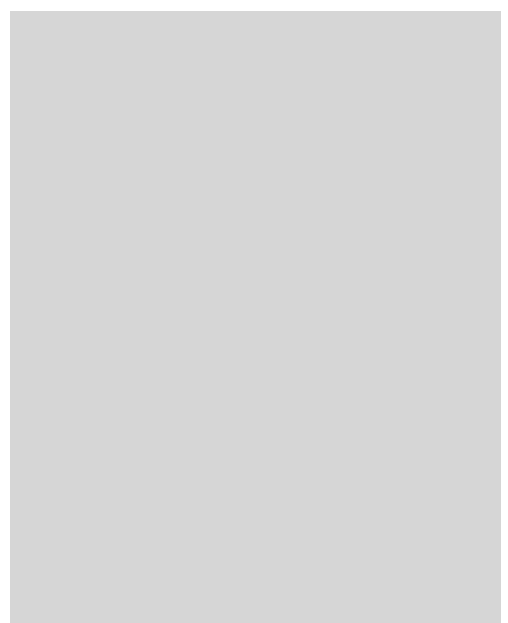

Fig. 2 - Page $10 \mathrm{du}$ Catalogue of the Philadelphia Photographic Salon, présenté du 24 octobre au 12 novembre 1898 aux Galleries of the Academy, à Philadelphie.

système de correspondances iconiques, plastiques ou sémantiques", la séquence se distingue par la logique temporelle qu'elle implique, par le développement chronologique qu'elle déploie, soit «une succession d'images dont l'enchaînement syntagmatique est déterminé par un projet narratif» ${ }^{21}$. Ainsi, au titre qui indique le développement oral discursif des Seven Words s'ajoutent les extraits des évangiles qui suggèrent un déroulement temporel et le cadre qui relie chaque étape.

Le rôle du texte et la relation étroite qu'il entretient avec l'image sont ici primordiaux. Le catalogue du Salon photographique de Philadelphie de 1898 liste les œuvres présentées par Fred Holland Day. Parmi celles-ci, The Seven Words apparaît en titre, suivi du deux-points de ponctuation et des sept paroles évangéliques numérotées (fig. 2). Cette indication tend à considérer chaque parole comme un sous-titre, soulignant ainsi l'autonomie de chaque image ${ }^{22}$. La séquence des Seven Words, formée de plusieurs épreuves développées spatialement en une frise légendée requiert en effet du spectateur de parcourir, de "lire" l'œuvre comme un texte, de gauche à droite, du début - "Père, pardonne-leur, car ils ne savent ce qu'ils font» (Luc 23:34) - à la fin - "Tout est consommé» (Jean 19:30) ${ }^{23}$. En outre, le format de la frise exige du regardeur qu'il se rapproche de

2I. Th. Groensteen, «Le réseau et le lieu», p. 123.

22. La même notation a été utilisée dans le catalogue de l'exposition de la PhotoSecession au National Arts Club de New York, en 1902. Voir W. I. Homer, Stieglitz and the Photo-Secession 1902, p. 37.

23. Kristin Schwain insiste sur la participation du spectateur qui, comme dans le cas des travelogues et du cinéma des premiers temps, doit "compenser une narration globale faite d'images discontinues fixes ou en mouvement». K. Schwain, «F. Holland Day's Seven Last Words and the Religious Roots of American Modernism», p. 42. 
l'œuvre et qu'à la première vue générale succède une découverte détaillée, déterminée par une logique narrative, de la première parole à la dernière.

\section{Le rôle du modèle}

Le déroulement temporel n'est pas uniquement à attribuer à l'encadrement et au texte, car les images elles-mêmes se chargent de traduire l'idée de durée. Les sept tirages constituent des autoportraits du photographe au visage émacié, aux cheveux longs et portant la couronne d'épines. Le traitement des épreuves aux effets vaporeux appréciés des pictorialistes estompe le haut du torse du Christ et insiste ainsi sur les attitudes et sur les expressions de son visage, faisant apparaître le sujet sur un fond clair où la croix est absente.

Fred Holland Day, en traduisant les paroles du crucifié au travers d'expressions de son visage, rend visible la parole. Le théologien suisse Lavater avait remarqué la carence descriptive de la physionomie du Christ dans les textes canoniques: «Il est surprenant que les évangélistes, et même saint Jean, le disciple favori du Seigneur, ne nous disent rien de sa personne, ni des traits de son visage " ${ }^{24}$. Le vide laissé par les évangiles dont les descriptions sont peu visuelles est comblé par The Seven Words. Day a sans doute également lu les grands traités physiognomoniques et le fameux ouvrage de Charles Darwin, L'Expression des émotions chez l'homme et les animaux (1872), dans lequel l'esprit et le corps de l'homme sont décrits comme un produit de l'évolution, thèse accompagnée de plusieurs photographies ${ }^{25}$. Tel un comédien sur scène, Day s'efforce, par le biais d'un mimétisme physique - le photographe a suivi un régime rigoureux le rendant rachitique - et au travers d'expressions du visage, d'incarner le personnage qu'il met en scène, fusionnant sa propre personne avec celle du Christ - l'identité de Day tend à s'effacer

24. J. C. Lavater, La Physiognomonie, p. 306.

25. K. Schwain, «F. Holland Day's Seven Last Words and the Religious Roots of American Modernism». 
derrière le rôle qu'il incarne ${ }^{26}$-, procédé qui ne manqua pas de créer la polémique ${ }^{27}$.

Les Seven Words peuvent renvoyer aux lanternes magiques très populaires au XIX ${ }^{\mathrm{e}}$ siècle et qui servaient aussi d'outil catéchétique ${ }^{28}$, mais l'œuvre est plutôt redevable du théâtre et des tableaux vivants ${ }^{29}$ appréciés de la photographie victorienne et en vogue aux Etats-Unis ${ }^{30}$. De plus, les représentations théâtrales du Mystère de la Passion ${ }^{31}$ données depuis le XVII ${ }^{e}$ siècle par les habitants d'Oberammergau en Bavière remportent un grand succès à l'époque et plusieurs artistes américains font le voyage pour les découvrir ${ }^{32}$. Fred Holland Day y assiste en 1890 et ne cache pas, une fois rentré aux Etats-Unis, l'influence que la production théâtrale allemande a eue sur son travail ${ }^{33}$.

La séquence physionomique souligne ainsi l'étroite relation entre le modèle photographique et l'acteur, Day donnant non seulement corps au Christ, mais assumant aussi ses mots, se faisant héritier des préceptes d'Horace qui stipulait que pour que l'artiste émeuve le public, celui-ci doit ressentir lui-même les émotions qu'il transmet dans son œuvre ${ }^{34}$.

26. Sadakichi Hartmann n'est d'ailleurs pas certain que ce soit bien Day qui ait posé à la place du Christ, l'auteur ayant de la peine à associer l'image du photographe et le rôle "auguste» qu'il s'attribue, même si la ressemblance physique plaide en faveur d'un autoportrait. S. Hartmann, "A Decorative Photographer: F. H. Day", in S. Hartmann, The Valiant Knights of Daguerre, p. 186-190. Cité aussi in V. Posever Curtis, J. Van Nimmen (eds), F. Holland Day, p. 69-73.

27. Sur les raisons du scandale, voir notamment: K. Schwain, «F. Holland Day's Seven Last Words and the Religious Roots of American Modernism»; J. Crump, "F. Holland Day: "Sacred" Subjects and "Greek Love" ".

28. Les récits, développés sur les plaques et projetés ensuite sur écran, combinent texte et image et déroulent une progression narrative segmentée, comparable à la fragmentation visuelle de la frise de Fred Holland Day. Sur les diverses influences visuelles de Day, voir K. Schwain, «F. Holland Day's Seven Last Words and the Religious Roots of American Modernism», p. 42.

29. Voir M. Poivert, «Aux origines de l'image performée».

30. V. Posever Curtis, «F. Holland Day».

3I. Voir Ch. Musser, "Les Passions et les Mystères de la Passion aux Etats-Unis (1880-1900)».

32. K. Schwain, «F. Holland Day's Seven Last Words and the Religious Roots of American Modernism», p. 43. Voir aussi Ch. Musser, "Passions and The Passion Play».

33. Voir Non signé, "Sacred and Modernized", The Boston Herald, 17 janvier 1899, p. 1, cité in The Photographic Work of F. Holland Day, p. 31.

34. " $\mathrm{Si}$ tu veux que je pleure, tu dois commencer par éprouver toi-même de l'affliction». Horace, Art poétique, v. 102-103, cité in R. W. Lee, Ut pictura poesis, p. 53. 


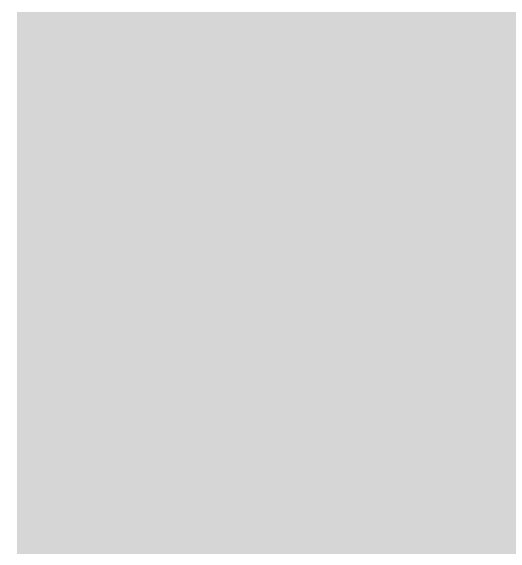

Fig. 3 - Nadar, Autoportrait tournant, ca 1865 .

\section{Expression, mouvement et durée}

En s'offrant le rôle du Christ, comment Fred Holland Day a-t-il cherché à traduire l'impression de temporalité, de déroulement du temps? La succession des autoportraits au cadrage identique traduit une durée, car même si le cliché photographique "opère une coupe dans l'espace et dans le temps, la juxtaposition des vues d'un même sujet, affecté de changements, renvoie aisément à une durée " ${ }^{35}$. D’ailleurs, Christian Metz, alors qu'il affirme l'incapacité de la photographie à raconter, évoque cependant la possibilité de narrer lorsqu'il y a juxtaposition d'images fixes:

Une photo isolée ne peut rien raconter; bien sûr! Mais pourquoi faut-il que par un étrange corollaire deux photos juxtaposées soient forcées de raconter quelque chose? Passer d'une image à deux images, c'est passer de l'image au langage ${ }^{36}$.

Cette multiplication des visages de Day, qui éveille l'impression de durée, rappelle les portraits en série réalisés dès les années 1860, et l'étonnant Autoportrait tournant (ca 1865, fig. 3) de Nadar, dans lequel le photographe est immortalisé sous douze angles différents, celui-ci faisant un tour complet sur lui-même. Cette "première forme de séquence» ${ }^{37}$ décompose le mouvement de Nadar et exige du spectateur, comme dans le cas de Day, qu'il complète les intervalles entre chaque image. Mais contrairement à l'autoportrait de Nadar, la séquence de Day propose le même point de vue sur le modèle et seule une légère évolution du corps est visible, de la souffrance à la mort. Georges Demenÿ, l'assistant d'Etienne-Jules Marey, à l'origine de la chronophotographie, expérimente en 1891 les mouvements de son visage lorsqu'il prononce des

35. D. Méaux, La Photographie et le temps, p. 129.

36. Ch. Metz, «Le cinéma», p. 63.

37. C. Chik, L'Image paradoxale, p. 62. 


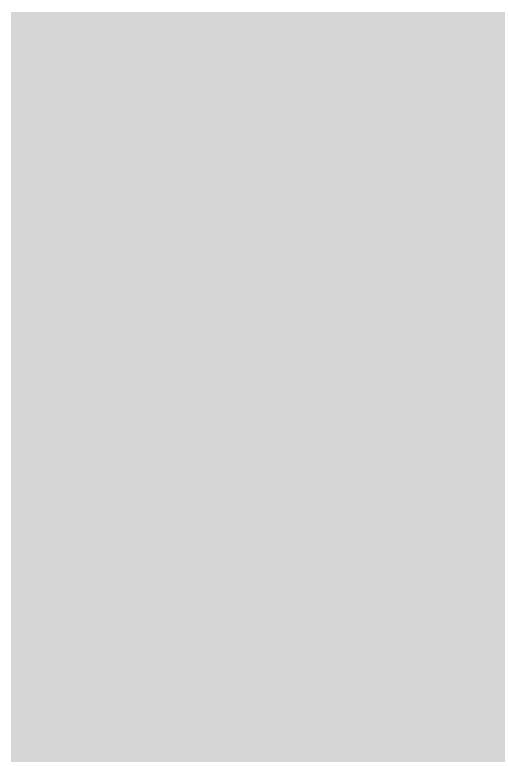

Fig. 4 - Georges Demenÿ, [Georges Demenÿ disant "Vive la France»], 1892. phrases devant son phonoscope (fig. 4). L'étude non plus d'actions - courir ou sauter à cheval -, telles qu'entreprises par Eadweard Muybridge dès 1878, mais d'expressions physionomiques impliquées par la parole, intègre "en somme, la narrativité » 38 , même si les recherches de Demenÿ se concentrent sur la "physiologie de la phonation et de la linguistique» 39 . Si ces expérimentations renvoient visuellement à l'œuvre de Day, leurs desseins diffèrent. Day ne vise ni l'animation de phases distinctes, ni l'étude des attitudes faciales lors de la prise de parole, mais la traduction visuelle, au travers des expressions du visage, des mots prononcés par le Christ sur la croix. En effet, Demenÿ décompose le mouvement découlant de l'élocution, alors que Day photographie des émotions, des états induits par des paroles.

En outre, la particularité des Seven Words réside dans le fait que les sept photographies condensent plusieurs heures vécues par le crucifié, durée elle-même réduite à quelques phrases lapidaires dans les quatre évangiles. D'une image à l'autre, les heures défilent - de la troisième heure à la neuvième heure ${ }^{40}$, mais le sujet, incapable de se mouvoir, impose au spectateur l'expérience de la durée du sacrifice. Comme le montre Kristin Schwain, le regard du photographe-modèle guide le spectateur et l'oriente dans son parcours de l'œuvre: les yeux du Christ qui fixent le haut et la droite dans la première œuvre, et le regard baissé vers la gauche dans la dernière épreuve, invitent le public à suivre un

38. Ibid., p. 98.

39. G. Demenÿ, Sur la chronophotographie, 1892, p. 29, cité in L. Mannoni, Georges Demenÿ, p. 43.

40. Voir Marc 15:25-26 à 15:33. 
mouvement circulaire ${ }^{41}$. Le mouvement du spectateur s'oppose ainsi brutalement à l'immobilité forcée du crucifié. L'œuvre de Day est-elle dès lors réellement narrative?

Choisissant la photographie La Sonnette (1934) de Robert Doisneau, dans laquelle un garçon lève le bras pour appuyer sur la sonnette d'une porte, alors que ses camarades s'enfuient déjà, donnant l'illusion que la course des jeunes préfigure le propre trajet de la figure principale, Philippe Marion déclare que cette image fixe «invite, incite au récit» ${ }^{42}$. L'effet cinétique de la photographie favorise l'impression qu'elle raconte une histoire, car la décomposition du mouvement au travers de plusieurs figures qui développpent virtuellement le futur déplacement du personnage principal, évoque une séquence temporelle. Ce processus que Philippe Sohet nomme "procédé de diffraction» ou de "cristallisation » ${ }^{43}$ est à l'œuvre dans la frise de Fred Holland Day dont l'«effet cinématique " ${ }^{44}$ est souligné, mais de manière différente: une seule figure déploie une succession de phases au sein de plusieurs images fixes. Il y a ainsi un agent affecté par une transformation, condition minimale pour qu'il y ait récit ${ }^{45}$. «Une séquence d'images (fixes ou mobiles) [...] [peut] raconter " ${ }^{46}$, certes, mais l'œuvre, si elle développe, il est vrai, le passage d'un état A au passage d'un état B - le Christ sur la croix souffrant au Christ mort - ne comporte aucune intrigue ou aucun nœud pour constituer une narration ${ }^{47}$. La particularité de l'œuvre de Day et sa complexité se mesurent aux textes qui se rapportent aux images. L'écrit ne forme pas un récit, mais réunit les phrases prononcées par le crucifié, rapportées au discours direct par les quatre évangiles et inscrites linéairement, soit chronologiquement, au-dessus des clichés. Le récit que Day représente exige un savoir supposé du public qui connaît le sujet

4I. K. Schwain, «F. Holland Day's Seven Last Words and the Religious Roots of American Modernism», p. 42.

42. Ph. Marion, "Les images racontent-elles?», p. 131.

43. Ph. Sohet, Images du récit, p. 60 sq.

44. "the cinematic effect». J. Crump, «F. Holland Day: "Sacred" Subjects and "Greek Love" ", p. 325.

45. G. Genette, Nouveau discours du récit, p. 14.

46. J.-M. Adam, Le récit, p. 9 sq.

47. La séquence narrative débute par une situation initiale, qui débouche sur un nœud, dont l'action ou l'évaluation aboutira au dénouement et à la situation finale. Voir J.-M. Adam, Le Texte narratif. 
abordé et, l'identifiant, peut reconstruire virtuellement la micro-scène qui se joue sous ses yeux ${ }^{48}$. Il faut bien l'admettre, sans les inscriptions, le spectateur serait bien incapable d'attribuer à chaque autoportrait la parole adéquate qu'elle traduit. Les attitudes sont peu variées et illustrent en réalité toutes le même état intérieur d'extrême souffrance. L'effet de temporalité qui émane de la frise de Day implique ainsi la participation active du spectateur et s'appuie sur le savoir collectif.

\section{Ut pictura poesis}

«[The photographer] no longer speaks the language of chemistry, but that of poetry. He quotes less Herschel than Pater ${ }^{49}$. Dans son texte "Is Photography an Art?», Fred Holland Day situe explicitement le statut du photographe du côté des poètes par opposition aux scientifiques. Il n'est pas anodin que l'auteur ait utilisé le champ lexical du langage - «speaks", "language», "quotes» - pour associer l'activité du photographe à l'œuvre de celui qui travaille la langue: le poète. Le photographe est un poète selon Day, qui renouvelle le fameux paragone de la Renaissance: la peinture et la poésie sont des sœurs ("sister arts»), leur nature, leur contenu et leur finalité sont similaires, seul leur moyen d'expression diverge ${ }^{50}$. La célèbre formule d'Horace ut pictura poesis (Ars poetica, v. 361), évalue la poésie qui «est comme la peinture» (ou «il en est de la poésie comme de la peinture»). La comparaison est reprise et inversée à la Renaissance: la parenté entre les deux arts est réduite au profit d'un discours qui valorise la supériorité de la poésie, prise comme modèle de la peinture ${ }^{51}$. Les auteurs de l'époque souhaitent libérer les arts plastiques de l'étiquette d'arts mécaniques et prouver la légitimité de la peinture et de la sculpture au sein des arts libéraux. L'énoncé resté

48. Sur la narrativité cognitive de la photographie, voir notamment J. Baetens, «Une photographie vaut-elle mille films?».

49. F. H. Day, "Is Photography an Art?", s.d. ca 1900, cité in V. Posever Curtis, «F. Holland Day», p. 300.

50. R. W. Lee, Ut pictura poesis, p. 15. Sur l'influence de cette formule au XIX siècle, puis au $\mathrm{XX}^{\mathrm{e}}$ siècle, voir $\mathrm{Ph}$. Junod, «Du péché de littérature chez les peintres».

5I. Voir Ph. Kaenel, «Livres, lectures, images, littératies». 
fameux, "la peinture est mentale " ${ }^{2}$ de Léonard de Vinci, gratifie la peinture d'un caractère fondamentalement intellectuel.

Les débats qui ont cours à la Renaissance au sujet de la peinture peuvent être rapprochés de ceux qui se produisent dès l'invention de la photographie, mais au caractère manuel des arts plastiques se substitue le procédé mécanique de la photographie. The Camera and the Pencil (1864) de l'Américain Marcus Root insiste sur le caractère artistique du procédé photographique: "sun-painting is not [...] a mere mechanical process; but, contrariwise, is one of the fine arts, and in its capabilities is, at least, the full equal of the others bearing this name» ${ }^{53}$. Fred Holland Day, dans la mouvance des pictorialistes américains, affirme l'autonomie de son médium, égal à la peinture ${ }^{54}$. Il insiste sur les moteurs du photographe, guidé par sa sensibilité et son esprit: «heart and intellect have collaborated " ${ }^{5}$. La genèse des Seven Words et des dizaines de clichés réalisés en 1898 sur la vie de Jésus relève d'une volonté de prouver que le médium photographique est capable de se libérer d'une représentation mimétique du réel et d'aborder les sujets allégoriques et religieux, au sommet de la hiérarchie des genres ${ }^{56}$. En affectant à la photographie le pouvoir de rendre visible le monde avec «une plus grande réalité et lucidité» ("greater reality and lucidity" ${ }^{57}$ ), employant le terme "lucidity» qui fait clairement référence à la "camera lucida», Day, influencé par le mouvement spirite de l'époque, affirme la double capacité du médium: la photographie enregistre le réel, mais paradoxalement, elle peut aussi

52. L. de Vinci, Traité de la peinture, MCM 16, C.U. $18 \mathrm{r}$.

53. En italique dans le texte. M. Root, The Camera and the Pencil, p. 25.

54. Notamment: "That the camera, properly guided, is capable of art - real art there is now no doubt." F. H. Day, "Art and the Camera", Camera Notes, vol. 1, n 2, oct. 1897, p. 27-28, cité in V. Posever Curtis, J. Van Nimmen (eds), F. Holland Day, p. $41 \mathrm{sq}$. "Although photography is scarce half a century old it is, if slowly, becoming a real factor to be reckoned with in Art.» F. H. Day, "Photography applied to the Undraped Figure", American Annual of Photography \& Photographic Times Almanac for 1898, New York, Scoville \& Adams, 1897, p. 186-197, cité in V. Posever Curtis, J. Van Nimmen (eds), F. Holland Day, p. 43-46.

55. F. H. Day, "Is Photography an Art?», s.d. ca 1900, cité in V. Posever Curtis, «F. Holland Day», p. 317.

56. Fred Holland Day explique: "I began to think of the possibility of making serious sacred work by means of the camera." Interview, p. 1, cité in The Photographic Work of F. Holland Day, p. 16.

57. F. H. Day, "Sacred Art and the Camera", The Photogram, fév. 1899, p. 37, cité in V. Posever Curtis, J. Van Nimmen (eds), F. Holland Day, p. 62. 
le percer et révéler ce qui se refuse à l'œil nu ${ }^{58}$. Il défend le potentiel fictionnel du médium et tente de légitimer la place de la photographie au sein des productions artistiques, capable d'exprimer le sacré. Day s'interroge d'ailleurs sur les mots employés pour définir une photographie:

Shall we not say that it [the picture] is not a work of art, because our vocabulary calls it a photograph, and because the man who made it, instead of holding between his fingers a bit of carbonized wood, has wielded a sunbeam ? 59

Homme de lettres ${ }^{60}$, Day qui sait manifestement également manier la plume, attribue au photographe une supériorité qu'il serait difficile de surpasser: le photographe travaille non pas avec des crayons ou des pinceaux, et encore moins avec des produits chimiques, le photographe dompte la lumière, il "manie les rayons du soleil» ${ }^{61}$. Rappelons que dans ce même texte, Day faisait de l'écrivain et critique anglais Walter Pater la référence des photographes et s'opposait à l'héritage d'un John Herschel. Cette prise de distance avec l'astronome et chimiste qui, grâce à ses recherches, permit d'améliorer les techniques photographiques dans la première moitié du XIX ${ }^{\mathrm{e}}$ siècle ${ }^{62}$, prouve que Day cherche à réduire l'aspect technique de la photographie. De plus, à la fin du XIX ${ }^{\mathrm{e}}$ siècle, Walter Pater était devenu "une figure culte aux Etats-Unis» ${ }^{63}$, dans le cercle des intellectuels de Boston, auquel appartient Fred Holland Day.

58. Sur les qualités médiumniques de la photographie, voir notamment C. Chéroux et. al., Le Troisieme ceil; J. Harvey, Photography and Spirit.

59. F. H. Day, "Is Photography an Art?", s.d. ca 1900, cité in V. Posever Curtis, «F. Holland Day», p. 317.

6o. James Crump souligne que les photographies de Day ont des sources «littéraires, artistiques et spirituelles». J. Crump, «F. Holland Day: "Sacred” Subjects and "Greek Love" ", p. 322.

6I. La formule renvoie à l'héliographie, «écriture du soleil» qu'emploie Nièpce et à la technique de l'héliogravure. Elle rappelle notamment l'album de photographies et de textes poétiques édité par Philip Henry Delamotte, The Sunbeam (1859) et l'ouvrage de John Towler, The Silver Sunbeam (1864). Sur la création du terme "photographie» - «écriture de la lumière» - et les discours sur l'invention du procédé, voir notamment F. Brunet, La Naissance de l'idée de photographie, p. 74-79.

62. Voir L. Schaaf, Out of the Shadows.

63. J. Crump, "F. Holland Day: "Sacred" Subjects and "Greek Love" ", p. 326. A propos des éditions américaines de l'ouvrage, voir la note 27 de l'article de Crump. 


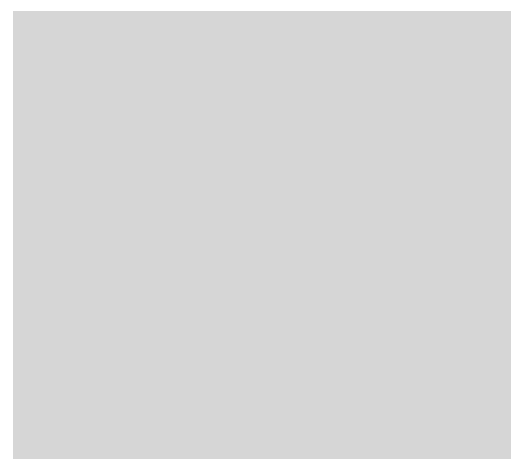

Fig. 5 - Détail du Laocoon d'Agésandros, Athanadoros et Polydore.
Son ouvrage, Marius the Epicurean (1885), qui célèbre un hédonisme liant la Grèce antique et le christianisme, a probablement influencé l'esthétique de Day, qui a traité non seulement de sujets chrétiens mais aussi de thèmes païens ${ }^{64}$.

Les Seven Words, par leur développement en frise évoquant une temporalité et par l'ajout des paroles issues des évangiles, tendent à contester Lessing qui, dans son texte majeur Laocoon (1766), distingue pour la première fois les deux "sister arts", qualifiant la poésie d'art du temps et octroyant l'espace aux arts plastiques ${ }^{65}$. Lessing estime que "la peinture ne peut exploiter qu'un seul moment de l'action et doit par conséquent choisir le plus fécond, celui qui fera le mieux comprendre l'instant qui précède et celui qui suit " ${ }^{66}$. L'auteur fait l'éloge de la sculpture du Laocoon ( $\mathrm{I}^{\mathrm{er}}$ siècle av. J.-C.), qui exemplifie l'instant fécond, défini comme un instant dramatique figé par le sculpteur et qui indique un procès en devenir, que l'œil du regardeur active par son imagination. Le photographe a en quelque sorte fragmenté l'«instant fécond " que loue Lessing et dévoilé les états successifs du crucifié avant son dernier souffle. Les expressions de son visage, bouche ouverte, tête penchée vers l'arrière, rappellent, il est vrai, l'attitude de la fameuse statue hellénistique (fig. 5-6).

\section{L'éloquence de la photographie}

Dans son compte rendu sur l'œuvre de Fred Holland Day, Sadakichi Hartmann emploie les adverbes "poetically» et "artistically» pour

64. A ce sujet, voir P. G. Berman, «F. Holland Day and His "Classical Models" ". A propos de l'influence de Walter Pater sur les intellectuels américains, voir J. Crump, «F. Holland Day: "Sacred" Subjects and "Greek Love" ".

65. G. E. Lessing, Laocoon.

66. Ibid., p. 120 sq. 


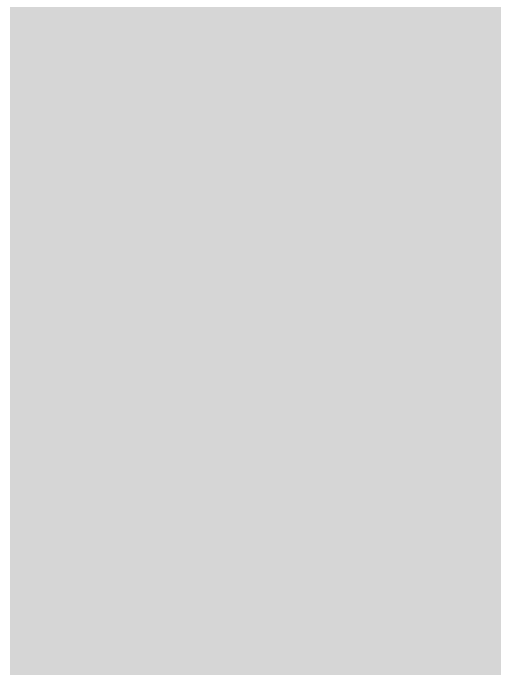

Fig. 6 - Fred Holland Day, [Christ with crown of thorns, frontal, head back], "Into thy hands I commend my spirit", The Seven Words, 1898.

décrire les ouvres de l'Américain, dont les arrière-plans «speak a language of their own, vibrant with rythm and melody" ${ }^{67}$. Le «lyrisme» 68 apposé aux photographies de Day qualifie parfaitement les Seven Words qui constituent une tentative de traduction des paroles relatées dans les évangiles par les expressions du visage.

Cette incarnation de la Parole est de portée à la fois personnelle et universelle. Fred Holland Day, jouant le rôle du Christ, identifie son statut à celui du créateur, à la manière de Dürer ${ }^{69}$, mais au visage hiératique de l'Autoportrait de 1500 s'oppose celui du crucifié, image de l'artiste martyr ${ }^{70}$. Le projet de Day est, il est vrai, audacieux et inédit en photographie ${ }^{71}$. Les Seven Words pourraient se comprendre comme une réponse à la fameuse formule de Simonide de Céos, citée par Plutarque dans De gloria Atheniensium, qualifiant la peinture de poésie muette et la poésie de peinture parlante (346f-347a). Pour les chrétiens, le sujet du Christ, Verbe fait chair, est littéralement mis en ouvre par Day qui incarne le crucifié et donne corps au verbal. En créant une relation de dépendance entre le texte et l'image et en déroulant le fil de ces visages qui se rapportent aux discours, les œuvres de Day sortent du mutisme auquel l'art plastique avait été longtemps

67. S. Hartmann, «Portrait Painting and Portrait Photography» [1899], p. 35-55, p. 46.

68. S. Hartmann, «Portrait Painting and Portrait Photography» [1899], p. 35-55, p. 46.

69. Voir Ph. Junod, «(Auto)portrait de l'artiste en Christ». Voir aussi N. Dietschy, "Ecce homo".

70. Voir notamment A. Sturgis, Rebels and Martyrs.

7I. Kristin Schwain cite notamment Julia Margaret Cameron, Louis Bovier ou Charles I. Berg. K. Schwain, «F. Holland Day's Seven Last Words and the Religious Roots of American Modernism». 
cantonné ${ }^{72}$. A la fin du XIX ${ }^{\mathrm{e}}$ siècle, à l'époque des prémices du cinéma (muet), alors que la photographie cherche à s'imposer parmi les divers types d'art, Fred Holland Day démontre avec les Seven Words que son médium n'a rien à envier aux autres formes de création et affirme, en recourant aux textes bibliques, l'autorité de la photographie ${ }^{73}$.

Les Seven Words se situent ainsi dans une zone frontière, proche du théâtre, des tableaux vivants et du cinéma. Le déploiement d'images fixes que l'œuvre met en scène tend à exprimer une séquence temporelle. Le personnage principal énonce lui-même l'épilogue: "Tout est consommé». En anglais, "It is finished» résonne à la fois comme les paroles du Christ et celles du photographe-modèle-acteur qui clôt la séquence. Le visage de la dernière photographie est d'ailleurs à moitié hors cadre: le modèle quitte l'espace de l'image alors que le personnage rend l'âme. Silence.

Nathalie Dietschy

Université de Lausanne

72. Les photographes des débuts sont restés silencieux, observe d'ailleurs François Brunet, qui cite le discours de François Arago en 1839 communiquant l'invention de Daguerre. F. Brunet, Photography and Literature, p. 88.

73. P. Geimer, "L'autorité de la photographie». 


\section{BIBLIOGRAPHIE}

Adam, Jean-Michel, Le Texte narratif, Paris, Nathan, 1994.

—, Le récit, Paris, PUF, 1999.

Art 39 Basel, cat., Ostfildern, Hatje Cantz Verlag, 2008.

Battens, Jan, "Une photographie vaut-elle mille films?», Protée, 34/2-3 (2006), p. 67-76.

Baetens, Jan, Bleyen, Mieke, "Photo Narrative, Sequential Photography, Photonovels", in Intermediality and Storytelling, ed. by Marina Grishakova, Marie-Laure Ryan, Berlin/New York, Walter de Gruyter, 2010, p. 165-182.

Barthes, Roland, La Chambre claire. Note sur la photographie, Paris, Gallimard, 1980.

Berman, Patricia G., «F. Holland Day and His "Classical Models": Summer Camp", History of Photography, 18/4 (hiver 1994), p. 348-367.

Brunet, François, La Naissance de l'idée de photographie, Paris, PUF, 2000.

—, Photography and Literature, London, Reaktion Books, 2009.

Chéroux, Clément, Fischer, Andreas, Canguilhem, Denis, Apraxine, Pierre, Schmidt, Sophie, Le Troisième aeil: la photographie et l'occulte, Paris, Flammarion, 2004.

Снік, Caroline, L'Image paradoxale: fixité et mouvement, Villeneuved'Ascq, Presses universitaires du Septentrion, 2001.

Crump, James, "F. Holland Day: "Sacred" Subjects and "Greek Love" ", History of Photography, 18/4 (hiver 1994), p. 322-333.

Dietschy, Nathalie, «Ecce homo: Jésus, l'artiste et l'homosexualité dans la photographie contemporaine", in Jésus en représentations, sous la dir. d'Alain Boillat, Jean Kaempfer, Philippe Kaenel, éd. par Nathalie Dietschy, Gollion, Infolio, 2011, p. 313-340.

Fanning, Patricia J., Through an Uncommon Lens: The Life and Photography of Fred Holland Day, Amherst, University of Massachusetts Press, 2008. 
Geimer, Peter, «L'autorité de la photographie. Révélations d'un suaire», Etudes photographiques, 6 (1999), p. 67-92.

Genette, Gérard, Nouveau discours du récit, Paris, Seuil, 1983.

Groensteen, Thierry, "Le réseau et le lieu: pour une analyse des procédures de tressage iconique dans la bande dessinée", in Temps, narration et image fixe, éd. par Jan Baetens, Mireille Ribière, Amsterdam, Rodopi, 2001, p. 117-130.

Hartmann, Sadakichi, The Valiant Knights of Daguerre. Selected Critical Essays on Photography and Profiles of Photographic Pioneers, Berkeley/Los Angeles/London, University of California Press, 1878.

Harvey, John, Photography and Spirit, London, Reaktion Books, 2007.

Homer, William Innes, Stieglitz and the Photo-Secession 1902, New York, Viking Studio, 2002.

JunoD, Philippe, "(Auto)portrait de l'artiste en Christ», in L'Autoportrait à l'âge de la photographie: peintres et photographes en dialogue avec leur propre image, éd. par Erika Billeter, Lausanne, Musée cantonal des Beaux-Arts, 1985, p. 59-76.

—, «Du péché de littérature chez les peintres: origine et portée d'un débat", Annales d'Histoire de l'art \& d'Archéologie, 16 (1994), p. 109-127.

Kaenel, Philippe, «Livres, lectures, images, littératies: d'une reproductibilité technique à l'autre (à partir de l'œuvre de Gustave Doré)", in Lire demain. Des manuscrits antiques à l'ère digitale, éd. par Claire Clivaz, Jérôme Meizoz, François Vallotton, Josef Verheyden, Lausanne, Presses polytechniques et universitaires romandes, 2012, p. 43-65.

Lavater, Johann Caspar, La Physiognomonie ou l'art de connaître les hommes d'après les traits de leur physionomie, leurs rapports avec les divers animaux, leurs penchants et leurs démarches, Lausanne, L'Age d'homme, 1979 (1777-1778).

LeE, Rensselaer W., Ut pictura poesis. Humanisme et théorie de la peinture: XV $V^{e}$ XVIII siècles, Paris, Macula, 1991.

Lessing, Gotthold Ephraim, Laocoon, Paris, Hermann, 1990 (1766).

Mannoni, Laurent, Georges Demenÿ. Pionnier du cinéma, Douai, éd. Pagine, 1997. 
Marion, Philippe, "Les images racontent-elles? Variations conclusives sur la narrativité iconique», Recherches en communication, 8 (1997), p. 129-148.

Méaux, Danièle, La Photographie et le temps, Aix-en-Provence, Publications de l'Université de Provence, 1997.

Metz, Christian, "Le cinéma: langue ou langage?", Communications, 4/4 (1964), p. 59-90.

Musser, Charles, "Les Passions et les Mystères de la Passion aux EtatsUnis (1880-1900)", in Une invention du diable? Cinéma des premiers temps et religion, sous la dir. de Roland Cosandey, André Gaudreault, Tom Gunning, Sainte-Foy/Presses de l'Université Laval, Lausanne/Payot, 1992, p. 145-186.

—, "Passions and The Passion Play: Theater, Film and Religion in America, 1880-1900", in Movie Censorship and American, ed. by Francis G. Couvares, Massachussetts, University of Massachussetts Press, 2006, p. 43-72.

The Photographic Work of F. Holland Day, cat. d'expo., Wellesley, The Wellesley College Museum, 1975.

Poivert, Michel, "Aux origines de l'image performée: la mise en scène photographique au XIX $\mathrm{X}^{\mathrm{e}}$ siècle», in Autour du symbolisme: Photographie et peinture au XIX $X^{e}$ siècle, éd. par Alain D'Hooghe, Bruxelles, Bozar Books, Palais des Beaux-Arts, 2004, p. 23-45.

Posever Curtis, Verna, «F. Holland Day: The Poetry of Photography», History of Photography, 18/4 (hiver 1994), p. 299-321.

Posever Curtis, Verna, Van Nimmen, Jane (eds), F. Holland Day. Selected Texts and Bibliography, Oxford, Clio Press, 1995.

Root, Marcus, The Camera and the Pencil. Or the Heliographic Art, New York, D. Appleton \& Co, 1864.

SchaAf, Larry, Out of the Shadows: Herschel, Talbot and the Invention of Photography, New Haven/London, Yale University Press, 1992.

Schaeffer, Jean-Marie, "Narration visuelle et interprétation", in Temps, narration et image fixe, éd. par Jan Baetens, Mireille Ribière, Amsterdam, Rodopi, 2001, p. 11-27.

Schwain, Kristin, «F. Holland Day’s Seven Last Words and the Religious Roots of American Modernism ", American Art, 1 (printemps 2005), p. 32-59.

Scotт, Clive, The Spoken Image: Photography and Language, London, Reaktion Books Ltd, 1999. 
Sohet, Philippe, Images du récit, Québec, Presses de l'Université du Québec, 2007.

Stoichita, Victor I., L'Instauration du tableau, Genève, Droz, 1999.

STURgIs, Alexander, Rebels and Martyrs: The Image of the Artist in the Nineteenth Century, London, National Gallery Company, 2006.

VIncI, Léonard de, Traité de la peinture, textes traduits et commentés par André Chastel, Paris, Calmann-Lévy, 2003.

Weitzmann, Kurt, Illustration in Roll and Codex, Princeton, Princeton University Press, 1970 (1947).

\section{Crédits iconographiques}

Fig. 1:

Epreuve au platine, $8,2 \times 33,9 \mathrm{~cm}$, Washington D.C., Library of Congress.

(C) The Louise Imogen Guiney Collection, Library of Congress, Prints \& Photographs Division, Washington D.C.

Fig. 2:

Philadelphia, The Pennsylvania Academy of the Fine Arts, 1898.

(C) The Metropolitan Museum of Art, New York.

Fig. 3:

Paris, Bibliothèque nationale de France, Département des Estampes et de la photographie.

(C) Bibliothèque nationale de France, Paris.

Fig. 4:

Fac-similé des photographies, légendé «Spécimen des photographies parlantes - Photographies des mots: vive la France!», publié in: Georges Demenÿ, "Les Photographies parlantes», La Nature: Revue des sciences et de leurs applications, 966 (1892), p. 312-315, p. 313.

Source: Conservatoire national des arts et métiers, Conservatoire numérique, http://cnum.cnam.fr

Fig. 5:

Sculpture en marbre, Musei Vaticani, Vatican. 
Fig. 6:

Epreuve au platine, Washington D.C., Library of Congress.

(C) The Louise Imogen Guiney Collection, Library of Congress, Prints \& Photographs Division, Washington D.C. 
